\title{
Discussion philosophique en classe et identité professionnelle du professeur d'école débutant
}

\author{
Michel Tozzi et Anne Lalanne
}

\section{(2) OpenEdition}

12 Journals

Édition électronique

URL : http://journals.openedition.org/trema/1406

DOI : 10.4000/trema.1406

ISSN : 2107-0997

Éditeur

Faculté d'Éducation de l'université de Montpellier

\section{Édition imprimée}

Date de publication : 1 janvier 2003

Pagination : 95-108

ISSN : 1167-315X

\section{Référence électronique}

Michel Tozzi et Anne Lalanne, «Discussion philosophique en classe et identité professionnelle du professeur d'école débutant», Tréma [En ligne], 20-21 | 2003, mis en ligne le 10 octobre 2010, consulté le 19 avril 2019. URL : http://journals.openedition.org/trema/1406 ; DOI : 10.4000/trema.1406

Ce document a été généré automatiquement le 19 avril 2019

Trema 


\title{
Discussion philosophique en classe et identité professionnelle du professeur d'école débutant
}

\author{
Michel Tozzi et Anne Lalanne
}

1 Deux formateurs coordonnent en IUFM une formation à cette innovation, débattant entre eux sur l'orientation à donner à des pratiques de terrain et de formation qui se cherchent...

\section{La problématique}

2 Des pratiques diversifiées de discussions philosophiques se développent actuellement en France à l'école primaire. Il est important d'accompagner cette innovation par la formation (initiale et continue) et la recherche.

3 Une première action, publiée dans le fascicule $\mathrm{N}^{\circ} 3$ des projets innovants réalisés en 2000-2001 à l'IUFM de Montpellier, visait à aider à mettre en œuvre puis à analyser ces nouvelles pratiques philosophiques, dans le cadre des parcours diversifiés des professeurs stagiaires de deuxième année. On y trouvait notamment :

- la question de l'expression et de l'écoute d'interrogations philosophiques d'enfants à l'école primaire ;

- une première typologie empirique des « réactions spontanées » des enseignants à ces questions ;

- un résumé des réticences et des objections rencontrées face à l'introduction de discussions philosophiques à l'école primaire ;

- un inventaire des types d'intérêt manifesté par ces pratiques ;

- le relevé des questions pédagogiques et didactiques posées pour ou à l'occasion de leur mise en œuvre. 


\section{impact de cette innovation sur la construction et l'évolution de l'identité professionnelle}

Michel Tozzi faisait alors lihypothèse, au vu des premières recherches, dun possible des enseignants du premier degré, en développant l'argumentation ci-après.

\section{Dans une posture traditionnelle, l'enseignant est le maître :}

- de la parole. Il décide s'il faut parler ou se taire, qui doit s'exprimer, à quel moment, si cette parole est pertinente ;

- du pouvoir. Il est institutionnellement dépositaire de l'autorité, responsable de l'ordre scolaire, garant des normes et des lois ;

- du savoir, reconnu compétent par sa formation et son recrutement.

En instituant un espace de débat, l'enseignant rompt avec cette posture :

- il donne un statut à la parole de l'élève, créditant chacun d'un droit d'expression ;

- son pouvoir peut être partagé, en délégant aux élèves certaines fonctions démocratiques (président de séance, responsable du temps de débat...) et cognitives (secrétaire de séance, synthétiseur, reformulateur...). La co-construction de règles discussionnelles, leur garantie par certaines rôles institués donne un style coopératif à la classe. L'éthique communicationnelle a des effets d'apaisement des conflits ;

- ce pouvoir ne cherche plus à s'imposer par un savoir incontesté. Le débat philosophique ouvre l'espace du discutable. Le maître ne pose plus aux élèves des questions dont il sait la réponse pour vérifier leurs connaissances. Ce sont les élèves qui (se) posent à eux-mêmes des questions, dont le maître est lui-même peu assuré. Celui-ci n'est plus le « sujet supposé savoir », mais un « sujet-sachant-douter».

7 C'est ce triple déplacement par rapport à une parole omniprésente, un savoir sûr de luimême, un pouvoir sans partage, qui peut redéployer une nouvelle identité professionnelle.

À partir d'une structure démocratique de l'échange, le nouveau maître institue une " communauté de recherche » (Lipman) ${ }^{1}$. Y prévaut un rapport non dogmatique au savoir. Celui-ci prend son sens en apparaissant comme une réponse cherchée et confrontée à partir d'une question que l'on se pose. Le maître ne tranche pas, n'apporte pas la réponse dernière. Il développe une culture de la question. Il veille aux exigences réflexives de la discussion philosophique: (s')interroger, savoir ce dont on parle (conceptualiser des notions) et si ce qu'on dit est vrai (argumenter rationnellement).

8 Écouter les élèves pour ce qu'ils (nous) disent, et non nous répondent, pour ce que leurs questions existentielles nous interpellent, canaliser leurs interactions vers des conflits socio-cognitifs en partant de leurs questions, c'est un changement radical. Dans cette articulation entre un rapport épistémologique ouvert au savoir et un rapport plus coopératif au pouvoir, dans cette démarche reposant sur l'énigme du questionnement et une éthique communicationnelle, s'ébauche peut-être le «maître de complexité »de demain...

9 Tel était pour Michel Tozzi l'objet de la formation proposée, qui affrontait le défi d'apprendre à animer des discussions philosophiques à des débutants :

- qui ne savent pas encore organiser des débats (gestion du groupe-classe en interaction sociale verbale, situation très difficile à anticiper dans une préparation) ;

- qui n'ont la plupart du temps que leurs souvenirs de classe terminale (manque de formation philosophique) ;

- qui ne peuvent pour l'instant guère trouver de classe ou d'école ayant ce genre de pratique, les tuteurs découvrant la pratique avec et par eux. 
Quelques présupposés sont d'après lui au fondement de la formation sur ces pratiques :

- philosophiques : le droit de philosopher dès le plus jeune âge ("Droit à la philosophie ", dit Derrida $\left.{ }^{2}\right) ;$ l'articulation en tension de pratiques démocratiques/philosophiques ;

l'« éducabilité philosophique » des enfants ;

- didactiques : une conception socio-constructiviste de la didactique de la philosophie ; le philosopher comme articulation de processus de pensée ; l'apprentissage possible du philosopher en discutant en groupe ; le concept de discussion philosophique, distingué d'autres pratiques sociales de références (le débat télévisé ou la discussion scientifique) ; un curriculumpossible de l'apprentissage de la discussion philosophique en fonction de l'âge ;

- pédagogiques : les concepts «d'espace public scolaire », de " démocratie scolaire », ou « dans l'acte d'apprendre »; l'articulation discussion philosophique - maîtrise de l'oral éducation à la citoyenneté - construction identitaire de l'élève.

\section{Le déroulement de la formation}

11 Dans le cadre d'un parcours personnalisé de 18 heures, la formation s'est articulée autour de trois axes principaux

\subsection{Premier axe : travailler sur les représentations des professeurs d'école}

\section{Questionnement des stagiaires en début de formation}

12 Nous avons, lors de la première séance, travaillé sur les questions des stagiaires. Celles-ci révèlent la prégnance de représentations sur la philosophie, provenant de l'enseignement reçu; sur la discussion, issues des pratiques sociales du débat; sur ce que pourrait être une discussion philosophique; sur la légitimité de cette pratique à l'école primaire et le rôle du maitre... On peut regrouper ces questions autour de trois pôles.

\subsubsection{Un pôle déontologique et éthique}

C'est le rapport à l'institution (programmes, réaction de l'inspecteur...) qui est interrogé : qu'en est-il de la légalité de ces pratiques ? Car la philosophie n'est pas une matière de l'école primaire. Va-t-elle être tolérée? Certaines questions sont des «analyseurs institutionnels»: faut-il prévoir un moment précis? Peut-on l'appeler atelier «philosophique »? Faut-il évaluer les compétences construites?

Le lien avec d'autres matières est évoqué, comme s'il fallait: une caution: le français, avec l'objectif de «maitrise de la langue orale» l'éducation civique, à travers l'apprentissage de «débats argumentés ». La demi-heure hebdomadaire d'oral prévue désormais est sécurisante!

Au-delà des réactions de l'institution, ce sont celles des familles qui émergent, car on touche à des sujets sensibles (religion, sexualité, politique). Faut-il ne pas parler de certains sujets, réservés à l'éducation familiale ? Ou au contraire faut-il à l'école parler des questions fondamentales? À une laïcité-neutralité s'oppose une laïcité de confrontation dans « l'espace public scolaire».

16 Au-delà d'un souci déontologique, on sent poindre un souci éthique, qui porte moins sur la légalité que sur la légitimité. En développant la rationalité très tôt, ne va-t-on pas 
contrarier l'épanouissement sensoriel, affectif, imaginaire de l'enfant (« Ils auront bien le temps d'être confrontés à ces problèmes angoissants ! »). Il est vrai que l'enfant se nourrit des contes pour se construire. Mais refuser d'entendre ses questions réelles (celle sur la mort arrive vers trois ans !) l'empêche de grandir, et le laisse seul face à ses questions !

Dans quelle mesure demander à un enfant de dire ce qu'il pense n'est pas une intrusion dans son monde intérieur? Mais si nos fantasmes sont intimes, nos idées ont-elles vocation à être tenues secrètes? Comment l'enfant pourrait-il vraiment apprendre à penser, c'est-à-dire à dialoguer avec lui-même, s'il ne dialoguait pas avec les autres ?

On ne voit pas comment on peut apprendre à philosopher sans secouer des certitudes : l'adulte prend peur de la situation anticipée, ou se trouve confronté au dilemme: la sécurité dans le conformisme, ou la mise au travail par l'interrogation? Comment permettre à l'enfant de douter dans un climat de confiance en soi, dans le groupe, dans le maître?

Cette activité doit-elle être imposée à tous, ou rester facultative ? Le volontariat semble respecter l'enfant, en lui donnant la responsabilité d'entrer dans l'activité ou d'en sortir. Mais d'un autre côté, ne prive-t-on pas l'enfant qui refuse d'une entrée privilégiée dans la réflexion, qu'il peut apprécier à terme?

\subsubsection{Un pôle didactique}

20 Un deuxième type de questions apparaît sur la spécificité disciplinaire de ces pratiques. Elles se disent "philosophiques». Cette discipline a laissé chez les stagiaires, anciens bacheliers, la représentation d'une matière où le cours magistral, des textes ardus et la forme dissertative sont irréalistes avec de jeunes enfants. De la philosophie en maternelle! D'emblée se pose la question de l'âge du philosopher. Les enfants sont pris dans l'émotion de leur vécu, incapables de décentration, imbibés des opinions familiales, gavés de télévision. Psychologiquement immatures, sans les assises de structures logiques ou de connaissances suffisantes, comment pourraient-ils exercer une pensée propre?

21 Ce questionnement renoue avec la conception cartésienne de l'enfance, lieu et temps du préjugé, obstacle à la réflexion personnelle. Peut-on postuler une «éducabilité philosophique de l'enfance »? Et si l'on a des activités réflexives avec les enfants, s'agit-il bien de philosophie? À supposer même qu'il s'agisse de pratiques " philosophiques ", ou plus modestement "à visée philosophique", doit-on introduire ce mot auprès des enfants? Quelques voix s'élèvent pour soutenir que cela peut les valoriser, puisque c'est une matière de " grands "...

Des représentations des stagiaires sur le " philosopher ", ce qui arrive en premier, c'est le type de sujet abordé : des questions touchant à la condition humaine comme l'amour et la mort, la liberté et le bonheur... Avec en fond le débat pour savoir si les enfants sont concernés de fait par ces problèmes, et doivent l'être en droit. D'autres se demandent quel genre de questions pourraient intéresser à coup sûr les enfants (« Avez-vous envie de grandir? Quelle différence entre un garçon et une fille, un homme et un robot, une fille et une poupée, etc. ?»). Lesquelles seraient utiles (« À-t-on le droit de tout faire ? À quoi servent les lois? Pourquoi dis-tu : «c'est pas juste !»).

D'où le statut de la question et de la réponse. Le primat de la question apparaît évident, parce que c'est ce qui met en recherche, exprime un rapport au savoir qui prend sens comme réponse aux questions que l'on se pose. 
On convient facilement qu'une question philosophique n'a pas de réponse unique. Certains se demandent si à certaines questions ("Qu'y a-t-il après la mort?») il y a vraiment des réponses, avec la tentation de conclure : s'il n'y a pas de réponse, à quoi bon encombrer la tête des enfants? On prend conscience aussi que selon la façon dont une question est formulée, on ne va pas répondre de la même façon. Par exemple : « La vie a-telle un sens? » ouvre la possibilité qu'elle pourrait ne pas en avoir, alors que « Quel est le sens de la vie? » implique de chercher le sens qu'elle peut avoir.

Le rôle du maître est interpellé. Partir des questions des enfants, c'est ouvrir un espace pour leur recherche. Comment comprendre ce qu'ils disent? Qu'est-ce qu'entendre philosophiquement une question d'enfant? Si c'est le maître qui questionne, l'enfant cherche à anticiper la réponse du maître, et se dessaisit d'une réflexion personnelle. Le questionnement du maître doit donc être formulé de façon ouverte. C'est lui qui peut, par son intervention questionnante, relancer le sujet, exiger d'aller plus loin. Mais s'il donne sa réponse (demandé souvent par les enfants eux-mêmes!), ceux-ci s'arrêtent de chercher!

L'idée paraît séduisante que les enfants confrontent leurs réponses pour progresser. Mais comment faire pour que la discussion soit philosophique, quand on sait déjà les difficultés à les faire discuter ensemble?

En l'absence de repères stabilisés sur ce qu'est l'apprentissage du philosopher, on comprend la question du «Comment vais-je savoir si ce que je fais est philosophique?». Il s'agit alors de clarifier les compétences philosophiques du maître et des capacités à développer chez les enfants.

Deux représentations émergent spontanément : philosopher, c'est interroger, nous dirons problématiser. C'est ensuite essayer de justifier. Cet accent sur l'argumentation pose peu problème, parce qu'elle est requise dans les programmes. Mais le caractère offensif de l'argumentation-objection l'emporte largement sur celle, plus philosophique, d'argumentation-fondement. Dans une "communauté de recherche", il s'agit plus de chercher avec que de lutter contre.

La capacité la plus occultée, peut-être parce qu'elle est la plus difficile pour les enfants, est la conceptualisation, la nécessité de définir le contenu des notions utilisées (l'amitié, la justice), ou de faire des distinctions conceptuelles («Être ami, c'est pas comme être copain ou être amoureux »).

C'est la clarification de ces trois processus de pensée (problèmatiser, conceptualiser, argumenter), et de la façon dont ils peuvent s'articuler dans l'unité et le mouvement d'une pensée impliquée, qui donne aux débutants les repères pour finaliser cette pratique, et l'analyser.

Ils comprennent alors pourquoi l'exemple, nécessaire à l'enfant pour rattacher une question abstraite à son vécu, est problématique pour une pensée philosophique, parce qu'il n'est pas, faute d'une généralité suffisante, un attribut du concept qui permettrait sa compréhension, mais tout juste une illustration; ni non plus un argument, parce qu'à tout exemple on peut en opposer un autre. Et pourquoi le contre-exemple est intéressant, concret en tant qu'exemple, tout en ayant statut d'argument puisqu'il détruit une induction trop hâtive. 


\subsubsection{Un pôle pédagogique}

31 «Le maître, il sait pas les réponses, et c'est nous les chefs de la parole !». Il y a là de quoi ébranler les représentations que l'on se fait d'un "Maitre ». La question de la faisabilité d'une discussion en grand groupe (a fortioriphilosophique), est évoquée. On voit le bénéfice escompté : apprendre à s'exprimer en public (capacité cognitivo-langagière), à écouter les autres (apprendre le respect), à tenter de les comprendre (se décentrer), etc.

«Tout cela est bien joli, mais les élèves ne vont-ils pas se disperser? Quelles règles pour ordonner la discussion? À quelles conditions seront-elles respectées?». Le souhait de travailler en petits groupes apparaît. Faut-il les composer en séparant les leaders, en groupes de niveaux pour tenir compte des différences de maturité, ou hétérogènes pour tirer vers le haut ceux qui peinent à abstraire?

«Comment mettre tout cela en place? ». Car les stagiaires doivent se couler en stage dans une organisation déjà là, avec des habitudes pédagogiques prises par les élèves. L'idée que discuter est un objectif, et pas seulement un moyen d'apprentissage, qu'un dispositif de discussion se met en place dans le temps est difficile à comprendre.

«Comment commencer la séance? Faut-il conduire un entretien de groupe en cumulant différentes fonctions (lancer la question, donner la parole, reformuler, recentrer, demander de préciser, faire des synthèses, etc.) ? Ou mettre en place une discussion, avec tables en rond, différents rôles (président de séance, responsable du micro, reformulateur, synthétiseur, journaliste, observateur, etc.)? Faut-il démarrer directement, ou s'appuyer sur un support? Comment terminer la séance ? Y a-t-il un rôle pour l'écrit, avant, pendant et après la séance?

Telle question peut arriver dès le départ, si le stagiaire est convaincu de l'intérêt de cette pratique. Sinon ce sont d'abord des résistances, des objections cognitives, institutionnelles, éthiques,. Mais beaucoup de questions apparaissent après une première expérience, quand les stagiaires confrontent la diversité des pratiques en fonction des classes, des écoles, des pédagogies du maitre en place, des âges, des entrées choisies dans l'activité...

\subsection{Deuxième axe : apport d'informations sur l'atelier de philosophie}

Anne Lalanne a choisi d'aborder la mise en pratique afin :

- d'informer les débutants sur des pratiques innovantes;

- de les sensibiliser à l'écoute des diverses interrogations des enfants ;

- $\quad$ - qu'elles émergent directement des disciplines : histoire : pourquoi s'intéresser au passé ? français : qu'est-ce qu'un nom ? math : peut-on tout compter? sciences : les choses sont-elles toujours comme elles le paraissent...?

- - qu'elles s'ancrent dans une quête de sens beaucoup plus générale : qu'est-ce que l'amitié ? peut-on tout savoir ? les animaux ont-ils une langue ou un langage? jusqu'où peut-on faire confiance à nos sens? qu'est-ce que la justice...?

- de les aider à réfléchir sur la façon de traiter ces questions dans une perspective philosophique avec des enfants dans le cadre de l'école? 
Pour une meilleure cohérence, Anne Lalanne a articulé son intervention autour de quatre points.

\subsubsection{Les objectifs de l'atelier}

Accompagner l'enfant dans ses premiers pas de sujet rationnel, c'est-à-dire : l'inviter à réfléchir, dans un travail intime de la langue et de la pensée, puisque c'est dans les mots que la pensée se cherche, en lui donnant l'occasion:

- de mettre en mots sa pensée, de tenter une formulation rationnelle de son expérience, en identifiant la source de ses représentations (d'où je sais ce que je sais) et en questionnant la valeur de cette source dans la confrontation dialoguée avec d'autres ;

- de faire usage de sa raison, en l'aidant à questionner l'opinion, à comprendre les enjeux de certaines représentations, à dépasser le sens commun pour s'ouvrir à l'universalité des idées et comprendre que sa recherche de sens n'est plus seulement la sienne mais aussi celle des autres et plus largement celle de tout être humain.

\subsubsection{Le rôle du maître}

Qu'est-ce qu'un maitre? Quel est son statut dans l'atelier? Selon Anne Lalanne, il s'agit moins de faire apparaître une nouvelle identité professionnelle rompant avec une posture dite traditionnelle que de comprendre quels sont les enjeux de son rôle dans la mesure où il n'est plus en face d'un savoir établi mais d'un savoir qui s'élabore. Dans la pratique d'un atelier de philosophie, il aura une fonction de guidage, d'accompagnement bien précis. Même s'il n'est pas en possession d'un savoir positif et transmissible, et se place lui-même dans une dynamique de réflexion, il n'en demeure pas moins compétent et reconnu comme tel, et donc garant de la progression de la réflexion du groupe et c'est à lui qu'incombe la responsabilité d'établir les liaisons conceptuelles tant que les enfants ne sont pas en mesure de les élaborer.

Il est nécessaire de distinguer la nature de ses interventions dans l'atelier en différenciant :

- l'animation, qui réside dans la gestion d'un dispositif donné, extérieur à tout travail sur le contenu;

- le guidage, centré spécifiquement sur le contenu et la progression de la réflexion collective, qui s'effectue à partir : de reformulations comprises comme mise à distance réfléchie de la parole et de son auteur, reprise de l'idée énoncée en vue de la relier aux enjeux du thème abordé ; de synthèses en tant que réelles analyses des divers arguments développés en vue de dégager plus clairement les enjeux parfois divergents voire contradictoires.

41 Tout ce travail de mise en forme des idées ne devient possible que grâce à un travail de préparation de l'atelier.

\subsubsection{La spécificité de la préparation du maître}

Reconnaître que le maître tient sa compétence de sa formation dans les disciplines qu'il est amené à enseigner à l'école signifie qu'il serait inconcevable et inacceptable qu'il s'engage dans la pratique d'un atelier de philosophie sans aucune formation en la matière. Il n'est pas nécessaire qu'il soit titulaire d'un CAPES de philosophie mais plutôt qu'il ait une formation de base (niveau Terminale minimum) réactualisée, au cours de formations, à travers des lectures lui permettant de conceptualiser à son niveau les 
thèmes et les questions qui vont être discutées lors des ateliers. Non pour faire une leçon mais pour être au clair sur des distinctions fondamentales, tant langagières que notionnelles, essentielles à la compréhension des enjeux d'une problématique donnée. Pour ce, il est intéressant de partir des interrogations d'enfants recueillies lors des stages, de repérer les différents types de questions posées et de les relier aux problématiques qui les sous-tendent. Ainsi, il devient possible de travailler le sens même de la question pour la faire évoluer en problème philosophique. À ce niveau, il existe des ouvrages de référence qui constituent une aide précieuse pour le maître, tel les livres de philosophie de Terminale. Il est certain que le niveau de préparation du maître ira bien au-delà de ce que les enfants pourront concevoir et c'est normal. Cependant, ce travail de préparation lui sera utile pour repérer dans le discours des enfants les arguments pertinents pour la réflexion et la construction d'une problématique, de partir de leurs formulations souvent maladroites afin de les aider à approfondir leurs idées et à les structurer. Seule une préparation cohérente et sérieuse permet au maître d'assurer son rôle de guidage sans lequel l'atelier de philosophie risque de tourner en conversation, démocratique certes, mais perdant toute visée philosophique.

On comprend ainsi pourquoi, les reformulations et les synthèses nécessaires à la cohérence de la réflexion sont de la responsabilité du maître. Cela ne veut pas dire que les enfants ne peuvent s'y essayer mais il faut entrevoir la difficulté de cette tâche pour eux : difficulté conceptuelle de comprendre la pertinence de ce qui est dit et capacité à le relier à différents aspects de la problématique travaillée.

Parce qu'il est important d'aider les enfants à travailler ces compétences, il semble fort dommageable que le maître délègue officiellement ces interventions spécifiques et capitales dans des dispositifs où il ne guide plus mais se place volontairement en retrait ou au même niveau que les enfants, revendiquant parfois la même ignorance. En quoi les enfants auraient-ils alors besoin de l'école pour participer à des discussions philosophiques dont ils seraient à même de mener seuls la réflexion? C'est parce que le maître sait guider qu'il est en mesure de les aider à travailler ces compétences progressivement dans le cadre des discussions.

Ici s'affirme une différence avec Michel Tozzi, qui tente d'articuler un dispositif démocratique de discussion avec des objectifs philosophiques.

\subsubsection{Les supports}

Ils sont nombreux et variés, notamment dans la littérature enfantine. Cependant, les interrogations des enfants eux-mêmes, les situations vécues peuvent servir de point de départ. Bref, toute remarque, tout étonnement peuvent être l'occasion de réflexion.

\section{La présentation de l'atelier aux enfants, aux familles, à l'institution}

À ce niveau, il est très important que l'enseignant sache exactement pour quelles raisons il s'engage dans la pratique d'un atelier de philosophie afin de le présenter correctement aux enfants d'abord, aux familles ensuite et à l'institution enfin.

L'atelier doit être présenté pour ce qu'il est : un moment de réflexion sur des thèmes, des questions de sens sans domaine circonscrit par avance. L'atelier de philosophie est un moment où l'on prend du temps pour penser son existence, son rapport aux autres et au monde, un moment où justement le temps est comme suspendu, durant lequel les enfants se posent enfin, physiquement (prendre son temps c'est savoir arrêter toutes les activités, 
se mettre sur le bord du chemin et pouvoir réfléchir sereinement) et intellectuellement car penser demande une certaine disponibilité de l'esprit, libéré des préoccupations matérielles immédiates. Dans une société où l'activisme est un critère d'existence, il est sans doute important de faire découvrir à l'enfant que prendre son temps n'équivaut pas à perdre son temps.

Cela revient donc à reconnaître qu'il ne peut être un moment où l'enfant se dit affectivement car nous sommes alors dans le domaine psychologique, où le groupe va discuter des difficultés rencontrées dans la vie de la classe; car cette optique suppose la mise en place de solutions réglant certains problèmes et nous ne sommes plus dans le domaine de la réflexion mais dans l'action (ce qui n'empêche que des situations vécues en classe peuvent servir de point de départ à un thème de réflexion philosophique, mais dans ce cas nous ne sommes plus dans la recherche de solution, on voit bien la différence des objectifs); ni un moment où l'on cherche à éduquer l'enfant à toute sorte de principes démocratique ou citoyen, car la réflexion n'est plus gratuite pour elle-même, mais se situe dans une optique idéologique et non plus philosophique. La philosophie n'éduque pas à [...], elle ne peut se réduire à une technique au risque de nier ce qu'elle est fondamentalement : un acte libre de la pensée, acte potentiellement subversif parce que fondamentalement libre. (Il n'est qu'à nous rappeler que c'est la démocratie athénienne qui a condamné à mort Socrate !).

Les enseignants doivent cette honnêteté aux enfants afin de ne pas les tromper sur l'acte même : la philosophie n'est pas un moyen en vue de [...] Réfléchir demande un effort et un investissement intellectuel, l'atelier en tant que lieu de pratique est le lieu de cet effort et il faut du courage pour oser penser. Il ne faut pas leurrer sur le contenu: la philosophie questionne tout, y compris elle-même, parce qu'elle revendique l'usage de la raison comme fondement à la pensée, qu'il n'y a pas de sujets tabous (à condition de les traiter dans une optique philosophique). De plus, même si le contenu n'est pas prédéterminé, qu'il se construit, tous les arguments ne se valent pas. Les limites de la raison ne débouchent pas sur le relativisme des opinions.

Ainsi, la pratique d'un atelier de philosophie à l'école sera-t-elle à l'abri de toute accusation de dérives affectives, psychologiques ou idéologiques.

\subsection{Troisième axe : la pratique d'une discussion en stage}

Michel Tozzi propose aux stagiaires de tester un dispositif ayant pour objectif d'articuler une structure démocratique du débat avec une visée philosophique. Les participants décident d'une question à débattre : peut-on faire de la publicité pour sa religion? Ils sont assis en demi cercle, et deux d'entre eux tiennent des rôles spécifiques : le « président de séance ", garant de la loi, distribue la parole, avec comme principe de la donner par ordre d'inscription, avec cependant la priorité à celui qui, voulant s'exprimer, ne l'a pas encore fait. Son rôle relève de l'organisation proprement dite. Un autre stagiaire est chargé d'être "synthétiseur » sur le fond, c'est-à-dire, à mi-parcours et à la fin de la discussion, de renvoyer au groupe les idées essentielles qui se sont dégagées. Quant à l'animateur de la formation, il prend le rôle de "reformulateur", ayant pour tâche de restituer au groupe ce qui se dégage au fur et à mesure, en mettant en relation les interventions entre elles et avec le sujet. Des observateurs sont chargés de prendre des informations sur les différents rôles exercés, et les processus de problèmatisation, de conceptualisation et d'argumentation à l'œuvre dans la séance. Dans une phase métacognitive, une analyse a 
lieu sur la façon dont le débat s'est déroulé, sur la manière dont les stagiaires ont vécu ces rôles, sur les exigences intellectuelles mises en œuvre.

\section{Analyse de la formation}

Deux temps forts sont apparus.

\section{Le travail effectué autour des travaux de Anne Lalanne}

Ceux-ci ont été l'occasion d'apporter des éléments d'éclaircissement par rapport à certaines interrogations exprimées en début de formation. L'analyse d'une pratique, quelle qu'elle soit, à travers l'aspect concret qu'elle expose pallie le manque d'expérience des débutants et leur offre la possibilité de sortir du domaine des fantasmes générateurs d'inhibitions. Rassurés, quelques-uns ont même tenté une ou deux séances lors du deuxième stage en responsabilité. Les échanges à ce propos ont été instructifs sur les aspects fondamentaux d'une formation et particulièrement sur l'importance de la préparation en amont des ateliers et sur le rôle de guidage du maître. Il ressort que les séances les mieux réussies ont été celles qui ont fait l'objet d'un travail de réflexion personnelle avant de se lancer avec les enfants. L'un d'entre eux a même pu comparer une séance faite spontanément avec une préparée : de la discussion qui s'en est suivie, il apparait que lorsque l'enseignant ne maitrise pas plus que les enfants certaines distinctions de base, il n'est pas en mesure de les aider à organiser leurs idées, que les interventions des enfants tournent vite en rond, faute de pouvoir baliser leur cheminement réflexif. Une sensation de confusion se mêle au sentiment d'inutilité d'une telle pratique. Par contre, l'impression d'avoir su accompagner les enfants dans la construction de quelques réflexions intéressantes est le résultat de leur capacité à les guider dans cette découverte de leurs pensées. Même s'ils ne se sentent pas encore pleinement au fait de certaines questions, l'expérience positive les conforte dans la volonté et la nécessité de travailler d'abord pour eux-mêmes des problématiques philosophiques. D'ailleurs, un enseignant cohérent peut-il prétendre à pratiquer un atelier de philosophie s'il n'est pas lui-même dans une dynamique de questionnement et de réflexion?

\section{La pratique d'une discussion philosophique avec un dispositif démocratique à visée philosophique avec Michel Tozzi}

La découverte d'un tel dispositif a fortement intéressé les stagiaires. L'analyse a montré qu'ils ont rencontré des difficultés à assumer correctement deux des rôles proposés :

1. Le président a été perturbé par le principe de priorité de parole. Si cette règle relève d'une volonté démocratique, elle peut rompre la construction de la pensée qui peut passer quelquefois par des mini-dialogues spontanés entre un ou plusieurs participants. D'autre part, les idées s'appellent. Attendre un tour de parole provoque certains retours en arrière qui font perdre le fil de la discussion. Celle-ci peut être vécue par le groupe comme bridée. Paradoxalement, le groupe s'est mieux investi lorsque le président, dépassé par la spontanéité des participants n'a plus géré les tours de parole. La discussion 
s'est révélée plus riche. D'où l'importance des reformulations et des synthèses pour ressaisir ce qui peut arriver dans le désordre.

2. Le synthétiseur : sa tâche est plus ou moins facilitée par le reformulateur. Mais il reste difficile d'opérer à chaud des liaisons entre les divers arguments lorsqu'on découvre le sujet en même temps que les participants. Cela suppose une capacité d'analyse immédiate et experte, qui suppose un apprentissage.

D'où des remarques des stagiaires :

- sur la capacité des enfants à s'acquitter de ces tâches complexes requerrant une faculté de recul et d'analyse nécessaires pour comprendre la pertinence de certains arguments ;

- sur le contenu inabouti de la discussion. Si les différents rôles sont tenus en classe par des élèves, il est difficile d'obtenir un guidage très cohérent, puisqu'ils sont tenus par des élèves en situation d'apprentissage.

Anne Lalanne souligne ici combien les objectifs sont déterminants: il ne suffit pas de poser une question, d'en discuter en argumentant pour faire de la philosophie: les politiques, les avocats le font tout aussi bien sans prétendre philosopher. Seul le guidage du maître permet aux élèves de construire leur réflexion et à terme rend possible une pensée autonome. Michel Tozzi pour sa part pense que c'est en s'entraînant à faire des reformulations et des synthèses que les élèves font des apprentissages intellectuels.

\section{Éléments de conclusion}

Un bilan du parcours personnalisé a été proposé à travers deux questions :

1. Est-ce que cette formation a modifié des éléments de la représentation que vous vous faites de votre métier?

60 La réflexion des stagiaires apparaît nuancée. Au lieu de parler d'une modification de leur représentation de leur métier, ils préfèrent l'analyser comme une lente maturation durant leur année de formation à l'IUFM. Maturation comprise comme une prise de conscience et une mise en mots de la complexité de l'acte d'enseigner. Si la formation propre au parcours personnalisé apporte un éclairage particulier, c'est principalement au niveau de l'articulation guidage du maître - activité de l'élève : alors qu'au début, la notion de guidage semblait négative, impliquant une certaine passivité des élèves, ils réalisent en fin de parcours l'importance et la spécificité de cet accompagnement vers l'autonomie des élèves, invités ici à construire leur pensée. C'est la difficulté de l'entreprise qui rend nécessaire un tel guidage. Du coup, réalisant l'importance de la qualité de leur intervention dans la construction du savoir chez l'élève, l'approche générale de leur métier en bénéficie.

61 2. Quelle a été pour vous l'utilité de cette formation? Elle se situe à plusieurs niveaux :

- la découverte d'une liberté d'initiative insoupçonnée dans le cadre institutionnel à travers une action innovante ;

- L'information sur l'organisation d'une discussion philosophique, se référant à des objectifs spécifiques. Plus généralement la conception de discussions pouvant avoir d'autres objectifs que philosophiques, notamment dans le domaine visant l'argumentation en sciences ;

- La prise de conscience que les disciplines scolaires peuvent ouvrir sur des problématiques philosophiques. Aspect essentiel pour aider les enfants à accéder au sens des apprentissages ;

- La comparaison de différentes pratiques et la mise à jour de leurs présupposés respectifs. 

Ce bilan a été accompagné de propositions sur les mises en situation souhaitables. Tout dispositif n'ayant de sens que par rapport à l'objectif philosophique de la discussion, il serait envisageable de les mettre en situation de préparer un thème pouvant être réalisé avec des enfants dans un cycle donné. La discussion pourrait alors être mieux analysée, tant dans sa forme qu'au niveau du contenu.

Si un tel type de formation, réservé pour l'instant à des volontaires, devait par la suite se généraliser, on voit tout l'intérêt d'une telle orientation pour travailler les attitudes de l'enseignant dans le sens d'une plus grande écoute des interrogations et des interventions des enfants, pour développer dans la classe une culture de l'interrogation, et transformer le fonctionnement traditionnel de la classe.

\section{BIBLIOGRAPHIE}

LALANNE A. : Faire de la philosophie à l'école. Paris, ESF, 2002.

TOZZI M. (Coord.) : L'éveil de la pensée réflexive à l'école primaire. Montpellier et Paris, CRDP Languedoc-Roussillon - CNDP - Hachette, 2002.

TOZZI M. (Coord.) : Discuter philosophiquement à l'école primaire - Pratiques, Formation,

Recherche. Montpellier, CRDP Languedoc-Roussillon.

TOZZI M. (Coord.) : Nouvelles pratiques philosophiques en classe, enjeux et démarches. Rennes, CRDP, 2002.

\section{NOTES}

1. LIPMAN M. : À l'école de la pensée. Bruxelles (Belgique), De Bœck, 1995.

2. DERRIDA J. : Du droit à la philosophie. Paris, Galilée, 1990.

\section{RÉSUMÉS}

Cet article rend compte d'une formation de philosophie avec les enfants pour des professeurs débutants à l'IUFM de Montpellier. Sont successivement décrits les objectifs poursuivis, les représentations des stagiaires, les apports d'information spécifiques, la pratique et l'analyse d'une discussion en stage entre enseignants. Puis sont présentés deux temps forts, ainsi qu'un bilan de la formation.

This article recounts a Montpellier Teacher Training College student teacher training course in philosophy instruction for Young children. Are described successively : the objectives pursued by 
the philosophy instruction course, the student teachers' mental representations on the subject, the contributions of specific information about philosophy for children, the conduct and analysis of a discussion on philosophy instruction held in teacher training class between the student teachers and their instructors. Afterwards, two of the training course's crucial moments are presented, as well as its appraisal.

INDEX

Mots-clés : discussion, école primaire, formation, identité professionnelle, philosophie pour enfants

Keywords : philosophy discussion, philosophy for children, primary school, professional identity, training session

\section{AUTEURS}

\section{MICHEL TOZZI}

Professeur des universités, directeur du Cerfee-Irsa et du département des sciences de l'éducation à Montpellier II

\section{ANNE LALANNE}

Maître - formatrice, IUFM de Montpellier 